\title{
Sum Rate of Linear Cellular Systems with Clustered Joint Processing
}

\author{
E. Katranaras, M. A. Imran and R. Hoshyar \\ Centre for Communication Systems Research CCSR \\ University of Surrey, UK \\ Email: e.katranaras@surrey.ac.uk
}

\begin{abstract}
In this paper we derive the sum rate of the uplink of a linear network of cells when clustered coordinated processing is adopted among the base stations in a generalised fading environment. Various cluster isolation schemes along with an interference allowance scheme are analysed and compared in terms of achievable sum rate with each other and to the optimum case of a system with central processor. Numerical results are produced for a real-world scenario.
\end{abstract}

\section{INTRODUCTION}

With increasing demand of spectrally efficient communication systems the search for the rate limits of uplink cellular systems gained momentum. First concrete findings in this regard ([1],[2],[3],[4]) assumed that all Base Station (BS) receivers cooperate in the uplink channel to jointly process the signals at a centralised receiver - termed as a hyper receiver via unlimited rate links. From the information theoretic system rate point of view, the joint processing of all the BSs in the system is optimal but this scheme is very hard to be implemented in real world. The large distances between BSs in most systems make it almost impossible to have a high rate connecting backbone among all BSs. Thus, the investigation of a clustered joint processing which decentralizes the joint decoding of all the users in the system poses a very interesting question to be answered. The concept of clustered multi-cell cooperative processing has attracted a lot of attention recently and is adopted in numerous investigations with some of them attempting to determine achievable rates or upper bounds ([5], [6], [7]).

In this work, we investigate and compare various clustering schemes. Clustering of cells in a system can be implemented in numerous ways. Frequency, time and space division schemes can be considered so as to isolate the clusters from each other. The principal focus of this research will be on finding the maximum achievable rate that each clustering scheme studied here can provide and to determine how close these rates are to the capacity of the overall hyper-receiver scheme. Moreover, an interference allowance scheme will be investigated where no isolation between clusters is considered. These findings will give important insights on the achievable bounds of cooperative multi-cell networks and determine if clustering is useful alternative to the hard to implement hyper-receiver scheme.

\section{SySTEM MODEL}

We investigate a similar system model as the one presented in [5] focusing on the uplink of a cellular system. We assume a linear cellular array and a network of cells where the BSs are uniformly distributed in a linear grid. Consider a system of $N$ cells divided into $M$ smaller networks (clusters of cells) each with $Q$ cells with $Q \ll N$. The cooperation among the base stations is limited only to the cells that belong to the same cluster and hence a Joint Processor (JP) in each cluster of cells jointly decode all the users of that cluster. The users are also uniformly distributed across the cells. We will assume that there is a maximum number of interference cell tiers $L$ for each BS after which the interference can be considered to be negligible. The widely accepted path loss and fading models that are presented in [8] are also employed here.

On the forthcoming analysis we will consider an average path loss approximation approach according to the one analysed in [9] for planar systems. The mean squared path gain for each of the $K$ users in a cell with radius $R$ which belongs in the $l^{\text {th }}$ tier of interference from the receiver of interest will be given by:

$$
\overline{\varsigma_{l}^{2}}=\frac{1}{2 R} \int_{-R}^{R} \frac{1}{\left(1+D_{l, k}(s)\right)^{\eta}} d s
$$

where $s$ is the random user distance from a BS and $\eta$ stands for the path loss exponent. Distance $D_{l, k}$ of a user in a cell in the $l^{t h}$ tier of interference from the receiver of interest is given by

$$
D_{l, k}(s)=2 R l+s
$$

\section{Sum Rate in Clustered System}

To facilitate sum rate analysis, we will assume uniformity between all clusters of cells. In that case our analysis can be performed only for a cluster and the results will be valid for all the clusters of the cellular system. The output vector of all the received signals in a cluster JP can be expressed in matrix form:

$$
\mathbf{y}=\mathbf{H} \mathbf{x}+\mathbf{z}
$$

where $\mathbf{y}=\left[y_{1}, y_{2}, \ldots y_{Q}\right]^{T}$ is the $Q \times 1$ received signal column vector, $\mathbf{x}=\left[\mathbf{x}_{1}{ }^{T}, \mathbf{x}_{2}{ }^{T}, \ldots \mathbf{x}_{Q}{ }^{T}\right]^{T}$ is the $Q K \times 1$ column vector of the transmitted signals of all the users in the cluster, with $\mathbf{x}_{q}=\left[x_{q, 1}, \ldots, x_{q, K}\right]^{T}$, denoting the concatenation of the 
transmitted signals from the $K$ users in cell $q, \mathbf{z}$ is the $Q \times 1$ column vector of noise and $\mathbf{H}$ is the overall $Q \times Q K$ cluster gain matrix given by:

$$
\mathbf{H}=\mathbf{\Sigma} \odot \mathbf{G}
$$

where $\boldsymbol{\Sigma}$ is a deterministic $Q \times Q K$ matrix that contains all the path gain coefficients and $\mathbf{G}$ is the $Q \times Q K$ matrix of all the fading coefficients of the channels in the cluster.

According to [10] the achievable rate of a cluster can be defined as the maximum of the mutual information between the transmitted and the received signals in the cluster over all distributions on the transmitted signals where each satisfy the power constraint $P_{k}$ :

$$
C_{\text {cluster }}=\max _{\forall p(x): \mathbb{E}\left[X^{2}\right] \leq P_{k}} I(\mathbf{x} ; \mathbf{y})
$$

Based on the information theory [11] we have that

$$
I(\mathbf{x} ; \mathbf{y})=H(\mathbf{y})-H(\mathbf{z})=\log \left(\frac{\operatorname{det}\left(\boldsymbol{\Lambda}_{\mathbf{y}}\right)}{\operatorname{det}\left(\boldsymbol{\Lambda}_{\mathbf{z}}\right)}\right)
$$

where $H$ stands for the entropy of the respective variable vectors and $\boldsymbol{\Lambda}_{\mathbf{y}}$ is the covariance matrix of the cluster output vector which for a fixed channel matrix $\mathbf{H}$ is described by:

$$
\boldsymbol{\Lambda}_{\mathbf{y}}=\mathbb{E}\left[\mathbf{y} \mathbf{y}^{\dagger}\right]=\mathbf{H} \mathbb{E}\left[\mathbf{x x}^{\dagger}\right] \mathbf{H}^{\dagger}+\mathbb{E}\left[\mathbf{z z}^{\dagger}\right]=\mathbf{H} \boldsymbol{\Lambda}_{\mathbf{x}} \mathbf{H}^{\dagger}+\boldsymbol{\Lambda}_{\mathbf{z}}
$$

Thus, the maximum achievable average per-cell rate can be given by:

$$
C=\mathbb{E}_{g}\left[\frac{1}{Q} \log \left(\frac{\operatorname{det}\left(\mathbf{H} \boldsymbol{\Lambda}_{\mathbf{x}} \mathbf{H}^{\dagger}+\boldsymbol{\Lambda}_{\mathbf{z}}\right)}{\operatorname{det}\left(\boldsymbol{\Lambda}_{\mathbf{z}}\right)}\right)\right]
$$

where the expectation is taken over all the fading realizations.

As $\Lambda_{\mathrm{x}}$ and $\boldsymbol{\Lambda}_{\mathrm{z}}$ are diagonal matrices of scalars, the important factor that has to be investigated in equation (8) is the $\mathbf{H H}^{\dagger}$ matrix. From the fact that the path gain and fading coefficients are uncorrelated with each other we have

$$
\mathbf{H H}^{\dagger}=(\boldsymbol{\Sigma} \odot \mathbf{G}) \cdot\left(\boldsymbol{\Sigma}^{T} \odot \mathbf{G}^{\dagger}\right)=\left(\boldsymbol{\Sigma} \boldsymbol{\Sigma}^{T}\right) \odot\left(\mathbf{G G}^{\dagger}\right)
$$

Each element of matrix $\boldsymbol{\Omega} \triangleq \mathbf{G G}^{\dagger}$ is the $K Q$ sum of random variables multiplied with the conjugate transpose of other random variables of the same distribution. When $K \rightarrow$ $\infty$ for every fixed $Q$, the horizontal dimension of $\mathbf{G}$ grows much faster than the vertical dimension. In this case the law of large numbers applies to each element of $\boldsymbol{\Omega}$

$$
\omega_{i, j} \cong K N^{2} \mathbb{E}_{g}\left[g_{n, k}^{q}\left(g_{\dot{n}, \dot{k}}^{\dot{q}}\right)^{*}\right], \forall i, j \in\{1, \ldots, Q\}
$$

where $g_{n, k}^{q}$ stands for the fading coefficient between a transmitter $k$ in cell $n$ and the BS of cell $q$. All the complex fading coefficients are normalized to unit power and when viewed as complex random processes are circularly symmetric i.i.d. Gaussian, stationary and ergodic. Hence, $\boldsymbol{\Omega}$ converges to a deterministic matrix equal to $\mathbb{E}_{g}[\Omega]$. Note that at the diagonal entries of $\Omega$ the product that takes place is:

$$
\mathbb{E}_{g}\left[g_{n, k}^{q}\left(g_{n, k}^{q}\right)^{*}\right]=\mathbb{E}_{g}\left[\left|g_{n, k}^{q}\right|^{2}\right]=1
$$

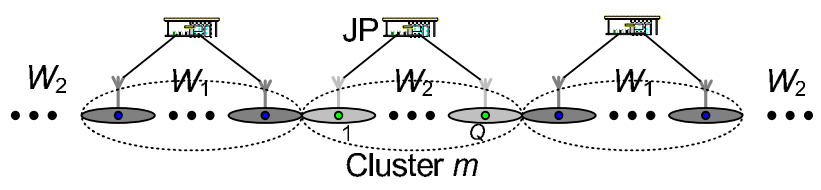

Fig. 1. Normal Spectral Division Isolation scheme

On the other hand, for the off-diagonal entries of $\Omega$ we have:

$$
\mathbb{E}_{g}\left[g_{n, k}^{q}\left(g_{n, \dot{q}}^{\dot{q}}\right)^{*}\right]=0
$$

implying that $\mathbb{E}_{g}\left[\mathbf{H} \boldsymbol{\Lambda}_{\mathbf{x}} \mathbf{H}^{\dagger}\right]$ converges to a diagonal matrix.

We use Jensen's inequality that provides an upper bound for the sum rate of the system:

$$
\begin{aligned}
\log \left(\operatorname{det} \mathbb{E}_{g}\left[\mathbf{H} \boldsymbol{\Lambda}_{\mathbf{x}} \mathbf{H}^{\dagger}+\boldsymbol{\Lambda}_{\mathbf{z}}\right]\right) & \geqslant \\
& \geq \mathbb{E}_{g}\left[\log \operatorname{det}\left(\mathbf{H} \boldsymbol{\Lambda}_{\mathbf{x}} \mathbf{H}^{\dagger}+\boldsymbol{\Lambda}_{\mathbf{z}}\right)\right]
\end{aligned}
$$

According to the above, with the number of UTs per cell growing large, the law of large numbers ensures that the upper bound presented in (13) is tight [3], [9]. Hence,

$$
C=\frac{1}{Q} \log \left(\frac{\operatorname{det} \mathbb{E}_{g}\left[\mathbf{H} \boldsymbol{\Lambda}_{\mathbf{x}} \mathbf{H}^{\dagger}+\boldsymbol{\Lambda}_{\mathbf{z}}\right]}{\operatorname{det}\left(\boldsymbol{\Lambda}_{\mathbf{z}}\right)}\right) \text { for } K \rightarrow \infty
$$

\section{Clustering Schemes}

\section{A. Normal Spectral Division Isolation Scheme}

The most simple division scheme for clustering is to divide the available frequency or time spectrum and let neighbouring clusters to operate in totally different frequencies/time slots. Since the system is considered to be uniformly clustered we assign here half of the available spectrum to each cluster with different resources assigned to adjacent clusters (Fig. 1).

According to the system model, the received signal at the BS antenna of cell $q$ in cluster $m$ is the sum of the transmitted signals from the users within the same cell and also from its maximum $L$ tier neighbouring cells appropriately scaled by the path gain and fading coefficients:

$$
y_{q}=\sum_{k=1}^{K} \varsigma_{q, k}^{q} g_{q, k}^{q} x_{q, k}+\sum_{\substack{q^{\prime}=1 \\ q^{\prime} \neq q}}^{q^{\prime}=Q} \sum_{k=1}^{K} \varsigma_{q^{\prime}, k}^{q} g_{q^{\prime}, k}^{q} x_{q^{\prime}, k}+z_{q}
$$

where $y_{q}$ and $z_{q}$ represent the received signal and the AWGN noise of $N_{0}$ power at the receiver of cell $q$ and $\varsigma_{q^{\prime}, k}^{q}$ stands for the path loss coefficient between a transmitter $k$ in cell $q^{\prime}$ and the BS of cell $q$. The variable $x_{q^{\prime}, k}$ represents the complex Gaussian inputs for a transmitter $k$ in cell $q^{\prime}$. It is assumed that each user has power constraint $P_{k}$, i.e. $\mathbb{E}\left[x_{q^{\prime}, k} \cdot x_{q^{\prime}, k}^{*}\right] \leq P_{k}$.

The maximum per-cell rate is achieved when all UTs are always allowed to transmit at their maximum transmit power constraint (wide band scheme presented in [3]). Thus, based on equation (14), the sum rate is given by:

$$
C_{n f t}=\frac{W_{1,2}}{Q W} \log \operatorname{det}\left(\mathbb{E}_{g}\left[\frac{P_{k}}{N_{0}} \mathbf{H H}^{\dagger}+\mathbf{I}_{N \times N}\right]\right)
$$

where $W_{1,2}=W / 2$ stands for either spectrum $W_{1}$ or $W_{2}$. 


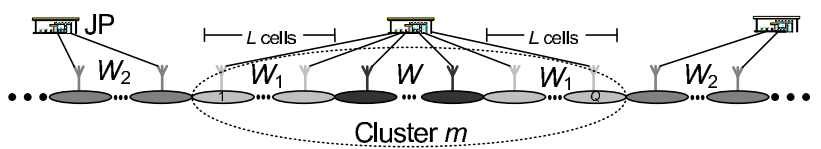

Fig. 2. Efficient Frequency Division Isolation scheme. When the $2 L$ edge cell users do not transmit at all, the scheme boils down to the Space Division Isolation one.

Taking into consideration the analysis about $\mathbf{H H}^{\dagger}$ matrix in the previous section and the cluster edge effects, the maximum achievable per-cell sum rate becomes:

$$
C_{n f t}=\frac{1}{2 Q} \log \prod_{i=0}^{Q-1}\left[1+\frac{K P_{k}}{N_{0}}\left(\sum_{j=0}^{\substack{j \leq L \\ i}} \overline{\varsigma_{j}^{2}}+\sum_{j=1}^{\substack{j \leq L \\ \varsigma_{j}^{2}}}\right)\right]
$$

Note that the above analysis presumes that $Q \geqslant L$.

\section{B. Efficient Spectral Division Isolation Scheme}

Although the normal spectral division scheme analysed above is very efficient for dividing the clusters from each other, it has the main disadvantage that every user is allowed to exploit only half of the available spectrum. A more efficient division scheme is investigated here. We allocate a part of the available spectrum only to cell users close to the edges of every cluster which may be able to cause interference to other clusters while letting all other users to use the full resources. Fig. 2 presents such a system where the BSs and their users at the $L$ cells from each side of the clusters are using spectrum $W_{1,2}$ to respectively receive or transmit. Using that division scheme, interference at the edges of each cluster is still avoided and, at the same time, the resources are used with the maximum possible efficiency by the system nodes. On the other hand, each JP can receive and process signals from the whole spectrum. The sum rate at a cluster $m$ though can be investigated separately for each spectrum.

The maximum per-cell rate will be given by

$$
C_{e f}=\frac{1}{W}\left(W_{1} C_{e f}^{(1)}+W_{2} C_{e f}^{(2)}\right)
$$

where $C^{(i)}$ is the sum rate achieved at spectrum $W_{i}$.

Following similar analysis as for the normal division scheme we can find $C_{\text {eft }}^{(1)}$ and $C_{\text {eft }}^{(2)}$ for $Q \geq 2 L+1$ as:

$$
\begin{aligned}
& C_{e f t}^{(1)}=\frac{2}{Q} \log \prod_{i=1}^{L}\left[1+\frac{K P_{k}}{N_{0}} \sum_{j=i}^{i+Q-2 L-1} \overline{\varsigma_{j}^{2}}\right]+ \\
& +\frac{1}{Q} \log \prod_{i=0}^{Q-2 L-1}\left[1+\frac{K P_{k}}{N_{0}}\left(\sum_{j=0}^{i \leq L} \overline{\varsigma_{j}^{2}}+\sum_{j=1}^{Q-2 L-1-i} \overline{\varsigma_{j}^{2}}\right)\right]
\end{aligned}
$$

and

$$
C_{e f t}^{(2)}=\frac{W}{W_{2}} C_{n f t}
$$

Hence, the per-cell sum rate will be given by replacing (19) and (20) in equation (18).

\section{Space Division Isolation Scheme}

Clustering of cells can be also achieved by using space instead of spectral division. Assume a model where the users at the $2 L$ edge cells will not transmit at all during a time slot (null cells). However, the BSs of these cells continue contributing on the clustered joint decoding process (Fig. 2 with $W_{1,2}=0$ ).

According to this scheme, following same steps as above, the maximum achievable per-cell sum rate will be given, again for $Q \geq 2 L+1$, by:

$$
C_{s d}=C_{e f t}^{(1)}
$$

\section{Interference Allowance Scheme}

Consider now a scheme where no isolation is considered between clusters, and users in all clusters are allowed to exploit the full resources allocated to the system. In that case, the edge cells of every cluster experience "inter-cluster" interference as their BSs will be interfered by transmitted signals from neighbouring cluster users.

Inter-cluster interference, since it is a sum of complex Gaussian inputs, it can be viewed as additional AWGN noise at the BSs and hence, it can be included in the $\boldsymbol{\Lambda}_{\mathrm{z}}$ covariance noise matrix in the general rate formula (8). It is obvious from there that the additional noise because of inter-cluster interference will decrease the achievable rate.

The received signal at the $\mathrm{BS}$ in cell $q$ is given now by:

$$
y_{q}=\sum_{k=1}^{K} \varsigma_{q, k}^{q} g_{q, k}^{q} x_{q, k}+\sum_{\substack{q^{\prime}=1 \\ q^{\prime} \neq q}}^{q^{\prime}=Q} \sum_{k=1}^{K} \varsigma_{q^{\prime}, k}^{q} g_{q^{\prime}, k}^{q} x_{q^{\prime}, k}+z_{q}^{\prime}
$$

where $z_{q}^{\prime}$ is the sum of the normal AWGN noise and the interfering complex Gaussian inputs from users outside the cluster of interest, e.g. $z_{q}^{\prime}=z_{q}+\sum_{\dot{q}} \sum_{k=1}^{K}\left[\varsigma_{\dot{q}, k}^{q} g_{\dot{q}, k}^{q} x_{\dot{q}, k}\right]$. Noise $z_{q}^{\prime}$ can still be considered AWGN with power given by

$$
\begin{aligned}
& \sigma^{2}=\mathbb{E}\left[z_{q, k}^{\prime} \cdot\left(z_{q, k}^{\prime}\right)^{*}\right] \\
& =N_{0}+\sum_{\dot{q} \notin[1, \ldots, Q]} \sum_{k=1}^{K} \mathbb{E}\left[\left(\varsigma_{\dot{q}, k}^{q} g_{\dot{q}, k}^{q} x_{\dot{q}, k}\right)\left(\varsigma_{\dot{q}, k}^{q} g_{\dot{q}, k}^{q} x_{\dot{q}, k}\right)^{*}\right]
\end{aligned}
$$

where $P_{e} \leq P_{k}$ is the power of the edge cell users signals. We consider different power constraint at the $2 L K$ edge cell users of each cluster, so as to investigate the effect of power control on the system sum rate.

The input covariance matrix will still be a diagonal matrix but not with equal diagonal elements:

$$
\boldsymbol{\Lambda}_{\mathbf{x}}=\operatorname{diag}[\overbrace{P_{e} \ldots P_{e}}^{L K} \overbrace{P_{k} \ldots P_{k}}^{(Q-2 L) K} \overbrace{P_{e} \ldots P_{e}}^{L K}]
$$




$$
\begin{aligned}
& C_{I}=\frac{2}{Q} \log \prod_{i=1}^{L}\left[1+K \frac{P_{k}\left(\sum_{j=L+1-i}^{L} \overline{\varsigma_{j}^{2}}\right)+P_{e}\left(\overline{\varsigma_{0}^{2}}+\sum_{j=1}^{L-i} \overline{\varsigma_{j}^{2}}+\sum_{j=1}^{i>1} \overline{\varsigma_{j}^{2}}\right)}{N_{0}+K P_{e}\left(\sum_{j=i}^{L} \overline{\varsigma_{j}^{2}}\right)}\right]
\end{aligned}
$$

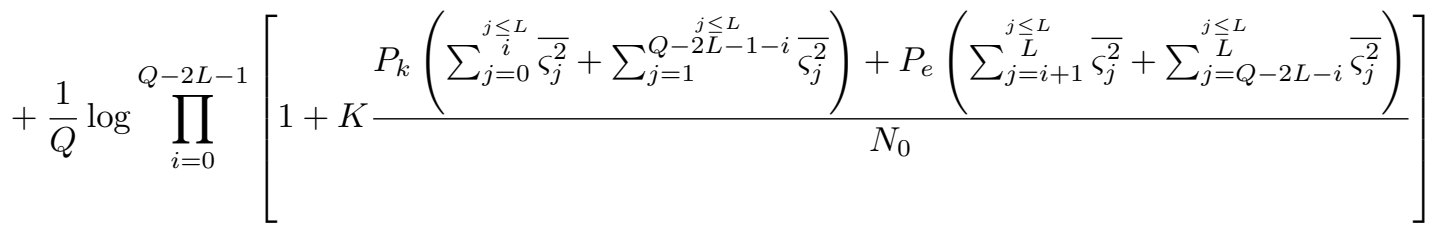

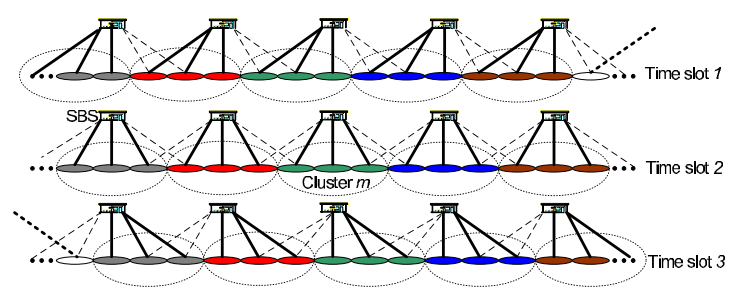

Fig. 3. Time Scheduling for a system with $Q=3$. The number of time slots needed to provide fairness will be exactly $Q$.

Furthermore the noise power matrix will be of the form:

$$
\boldsymbol{\Lambda}_{\mathbf{z}}=\operatorname{diag}[\overbrace{\sigma^{2} \ldots \sigma^{2}}^{L} \overbrace{N_{0} \ldots N_{0}}^{(Q-2 L)} \overbrace{\sigma^{2} \ldots \sigma^{2}}^{L}]
$$

Based on the above and considering equation (14) for large number of users per cell and for $Q \geq 2 L+1$, the maximum achievable per-cell sum rate will be given by (25). Note that a circular array model [2] can be considered to avoid the edge effects.

\section{E. Time Scheduling. A solution for Fairness}

In a static linear cellular clustered system the edge cells from each cluster will be able to achieve less rate than the central cells. For a balanced system with equally served cells, a Time Scheduling scheme may be considered where the links between the JPs and cells change dynamically with time (Fig. 3 ). For this, each JP in that case needs to be linked with $Q^{\prime}=2 Q+1(>Q)$ BSs but eventually all the cells will get equally all the possible states within the cluster during a period of time. In that way, it will be possible to achieve fairness in the sense of cell rate.

\section{Numerical Results}

For interpreting the information theoretic results into realworld systems we assume the environment described in [8] and the respective system modelling parameters are used. According to this system we choose a very dense system scenario $(\eta=2, \mathrm{ISD}=100 \mathrm{~m}, K=100$ users per cell with $P_{k}=200 \mathrm{~mW}$ each) and we investigate the cell rate for the case where each cluster is formed by a single cell. By this we intend to estimate the impact of the maximum tiers of interference considered on the per-cell rate. Fig. 4 illustrates
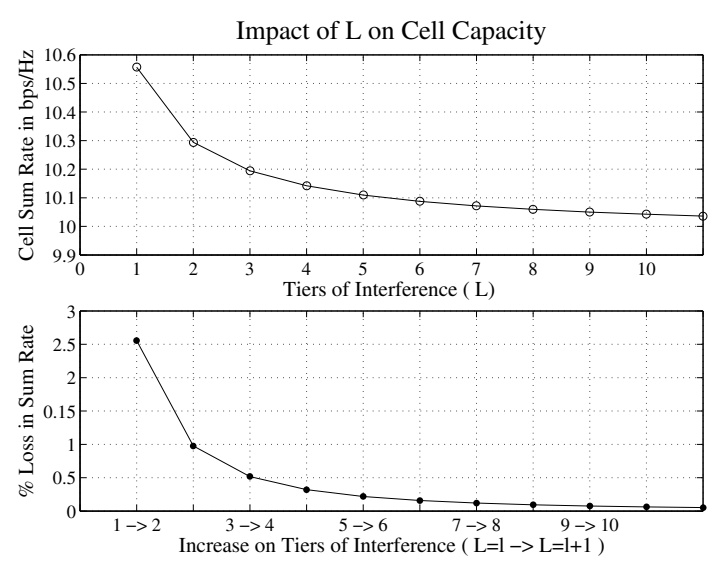

Fig. 4. Impact of maximum interference tiers considered $(L)$ on cell rate.

the sum rate of the single cell cluster versus the tiers of interference considered according to

$$
\breve{C}_{I}=\log \left(1+\frac{K P_{k} \overline{\varsigma_{0}^{2}}}{N_{0}+2 K P_{k}\left(\sum_{j=1}^{L} \overline{\varsigma_{j}^{2}}\right)}\right)
$$

It can be seen that in the linear model with these specific parameters there is no point of considering more than one tiers of interference as the loss in cell rate because of more tiers is negligible $(<3 \%)$. Note that the maximum achievable per-cell rate for a hyper-receiver using system is given by

$$
\hat{C}_{I}=\log \left(1+\frac{K P_{k}}{N_{0}}\left(\overline{\varsigma_{0}^{2}}+2 \sum_{j=1}^{L} \overline{\varsigma_{j}^{2}}\right)\right)
$$

and with the system parameters used above this reaches to $\hat{C}_{I} \simeq 26.6 \mathrm{bps} / \mathrm{Hz} /$ Cell. Nevertheless, $L=3$ was considered to illustrate the results in figures 5 and 6 . The results have been verified by running Monte Carlo simulations to generate random fading coefficients for various system snapshots. The simulation rates are obtained by finding the average over a large number of fading snapshots using equation (8).

In Fig. 5 a comparison is provided among the various clustering schemes, the interference allowance (with $P_{e}=P_{k}$ ) and the hyper receiver scheme for two different scenarios. In 


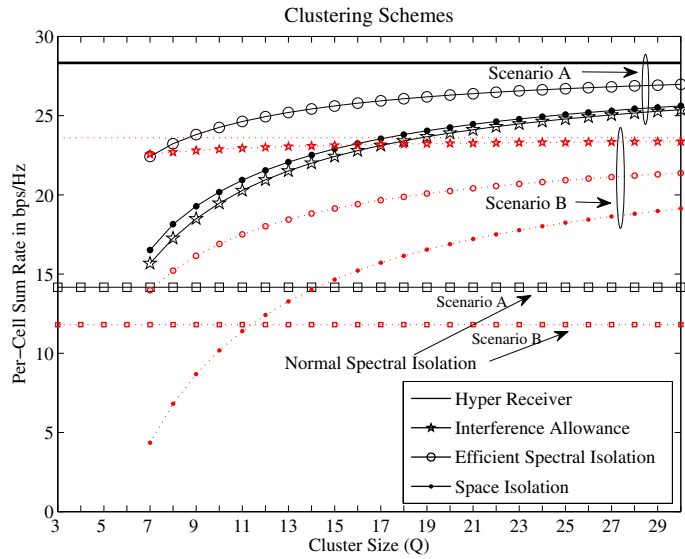

Fig. 5. Comparison of the various clustering schemes.

Scenario A, a dense system is considered with $\eta=2.5$, InterSite Distance (ISD) of $200 \mathrm{~m}, K=100$ users per cell with $P_{k}=200 \mathrm{~mW}$ each. We observe that the efficient spectral isolation scheme is preferable for this case. For a more sparse system (Scenario B: $\eta=3$, ISD $=2 \mathrm{Km}, K=100$ users per cell with $P_{k}=100 \mathrm{~mW}$ each) the Interference allowance scheme becomes optimal when cell clustering is implemented and reaches close to the maximum achievable rate. It is also noted that the normal spectral isolation scheme achieves almost half of the maximum achievable rate while the space isolation scheme is always suboptimal to the efficient spectral one.

In Fig. 6 the effect of power control at the edge cell users of clusters on per-cell rate is investigated for the Interference allowance scheme. It is clear that there is no point of applying power control in sparse systems while in dense system we have a marginal improvement. Finally, in both figures 5 and 6 we can observe that if there is no isolation scheme available, it is preferable to have small clusters when our system is sparse (as inter-cluster interference is negligible in that case) while larger cluster size is needed to achieve higher rates in a dense system.

\section{CONCLUSION}

The information theoretic sum-rate of the uplink of a linear clustered cellular system that uses cooperation at the multiple BS receivers of each cluster has been derived here for various cluster isolation schemes. It has been observed that even with small cluster sizes, large portion of capacity can be achieved. Low path loss exponent, low transmitting power and large cell size lead to isolation among clusters in which case the cluster isolation schemes are suboptimal and the interference allowance scheme is preferred and vice versa. Power control at edge cell users when allowing inter-cluster interference is meaningful only in dense systems. The ideas are readily extendible to the more realistic planar system and this poses a very interesting question to be further analysed in our future approach.

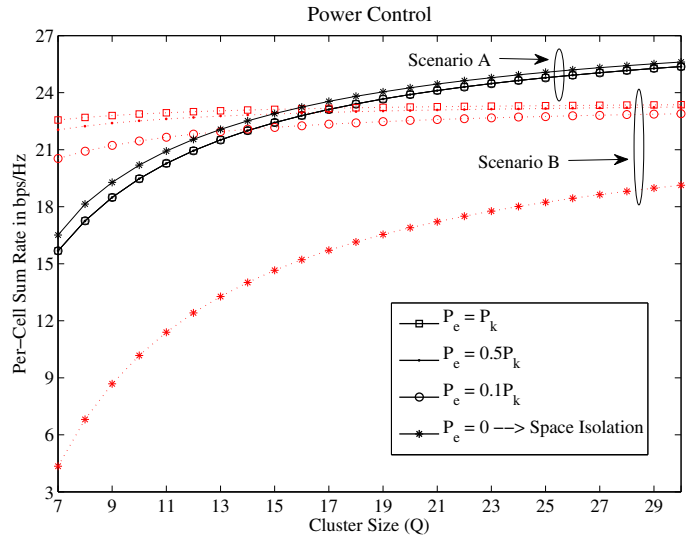

Fig. 6. The effect of power control at the edge cell users of clusters on per-cell rate.

\section{ACKNOWLEDGMENT}

The work reported in this paper has formed part of the "Fundamental Limits to Wireless Network Capacity" Elective Research Programme of Virtual Centre of Excellence in Mobile \& Personal Communications, Mobile VCE, www.mobilevce.com. This research has been funded by the following Industrial Companies, Members of Mobile VCE BBC, BT, Huawei, Nokia Siemens Networks, Nortel, Vodafone. Detailed technical reports on this research are available to staff from these Industrial Members of Mobile VCE.

\section{REFERENCES}

[1] A. Wyner, "Shannon-theoretic approach to a gaussian cellular multipleaccess channel," IEEE Transactions on Information Theory, vol. 40, no. 6, pp. 1713-1727, Nov. 1994.

[2] S. V. Hanly and P. A. Whiting, "Information-theoretic capacity of miltireceiver networks," Tellecommun. Syst., vol. Vol. 1, pp. pp. 1-42, 1993.

[3] O. Somekh and S. Shamai, "Shannon-theoretic approach to a gaussian cellular multiple-access channel with fading," IEEE Transactions on Information Theory, vol. 46, no. 4, pp. 1401-1425, July 2000.

[4] N. A. Letzepis, "Gaussian cellular muptiple access channels," Ph.D. dissertation, Institute for Telecommunications Research, University of South Australia, December 2005.

[5] M. Bacha, J. Evans, and S. Hanly, "On the capacity of mimo cellular networks with macrodiversity," in Proc. 7th Australian Communications Theory Workshop, 2006, 1-3 Feb. 2006, pp. 105-109.

[6] A. Vanelli-Coralli, R. PAdovani, J. Hou, and J. Smee, "Capacity of cell clusters with coordinated processing," in USCD Inaugural workshop on Information theory and its applications, La Jolla, CA, 2006.

[7] O. Somekh, B. M. Zaidel, and S. Shamai, "Spectral efficiency of joint multiple cell-site processors for randomly spread ds-cdma systems," IEEE Trans. Inf. Theory, vol. 53, no. 7, pp. 2625-2637, July 2007.

[8] E. Katranaras, M. Imran, and C. Tzaras, "Framework to compare the uplink capacity of the cellular systems with variable inter site distance," In Proc., IEEE PIMRC 08 International Workshop on Efficiency, 2008.

[9] — , "Uplink capacity of a variable density cellular system with multicell processing," Accepted for publication in IEEE Transactions on Communications, 2008.

[10] C. E. Shannon, "A mathematical theory of communication," Bell Systems Technical Journal, vol. 27, pp. 379-423, 1948.

[11] T. M. Cover and J. A. Thomas, Elements of Information Theory. John Wiley \& Sons, New York, 1991. 